\title{
colaboraciones
}

\section{EL AUTO-DREN: UNA NUEVA PROTECCION PARA LOS REVESTIMIENTOS DE CANALES}

\author{
(AUTO-DREN: A NEW PROTECTION FOR PIPES COATING)
}

Federico Moreno Nieves, Ing. de Caminos

\section{RESUMEN}

Las consideraciones que siguen se dedican al fenómeno de rotura por subpresión en canales y depósitos enterrados. Tras la descripción del proceso de rotura y sus circunstancias, se propone una solución simple y eficaz para obviar el problema.

Queremos agradecer a los Ingenieros responsables de Construcción y Conservación de las Confederaciones Hidrográficas su postura de colaboración y transmisión de su experiencia que tanto nos ha posibilitado el conocimiento de la problemática que a continuación tratamos.

\section{SUMMARY}

The following considerations are devoted to the study of buried pipes and tanks fracture by uplift. After the description of the fracture process and its circumstances, it is proposed a simple and efficient solution to avoid the problem.

We want to thank the Construction and Preservation Engineers of the Hydrographic Confederations for their collaboration and for bestowing their experience that have enabled us to know the problem we describe.

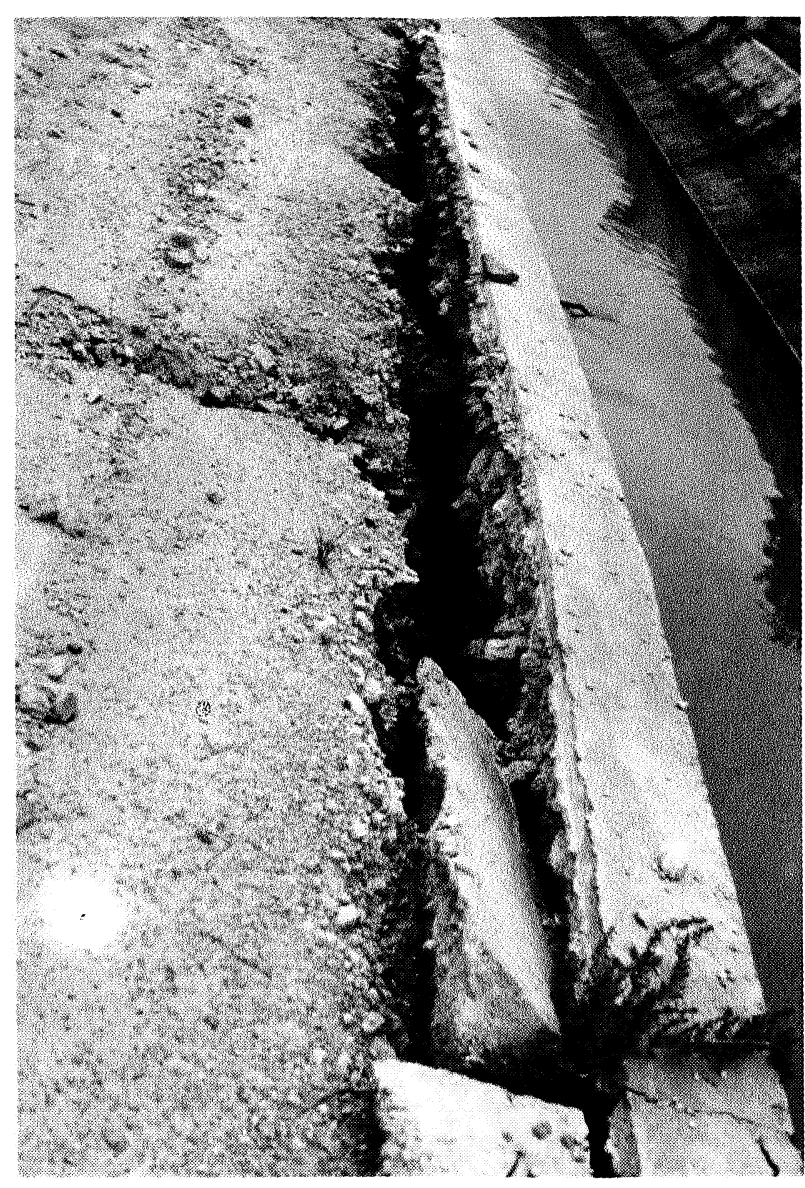

Las averías por fisuración en canales y depósitos son motivo de pérdidas por fugas y de un coste adicional de la explotación por reparaciones, amén de otras consecuencias como desfases accidentales en los riegos y suministros que, aunque paliadas en gran proporción por la coordinación y atención de los responsables de los correspondientes servicios, son inevitables en algunas ocasiones.

Aunque no existen aforos exactos para evaluar los caudales perdidos o los costes de reparaciones que, en muchas ocasiones, quedan englobados en los de limpieza u otros trabajos de conservación, la extrapolación de mediciones realizadas en tramos concretos y aforos locales, sitúan las cifras para el total de España, entre 300 $\mathrm{Hm}^{3}$ y $1.500 \mathrm{Hm}^{3}$ de agua que entra en las redes de distribución y no llega a su destino, anualmente. $Y$ entre 3.000 y 6.000 millones de pesetas, también anuales, el coste real de reparaciones de tales averias en la red de canales y acequias. Se trata, pues, de un coste económico importante.

En las consideraciones que siguen, se analizan el efecto de flotación de las secciones revestidas en canales y depósitos enterrados y sus consecuencias en la obra. Asimismo, se describe el fenómeno de saturación de los conductos drenantes que, en muchos casos, se disponen bajo dichas obras. 
Finalmente se proponen soluciones que creemos útiles tanto para evitar la presentación de estos problemas como para corregir los efectos nocivos en los casos en que tal situación ya se ha producido; soluciones con las que quisiéramos aportar algo a las ya existentes.

\section{DESCRIPCION DEL FENOMENO}

La búsqueda de materiales y tecnologias adecuadas a la reparación de canales, nos ha permitido el conocimiento directo de una cantidad importante de tramos averiados por rotura del revestimiento, en terrenos de caracteristicas muy variadas. Esta muestra nos parece suficientemente representativa para clasificar las roturas en función de su morfologia, en tres grandes grupos (Fig. 1):
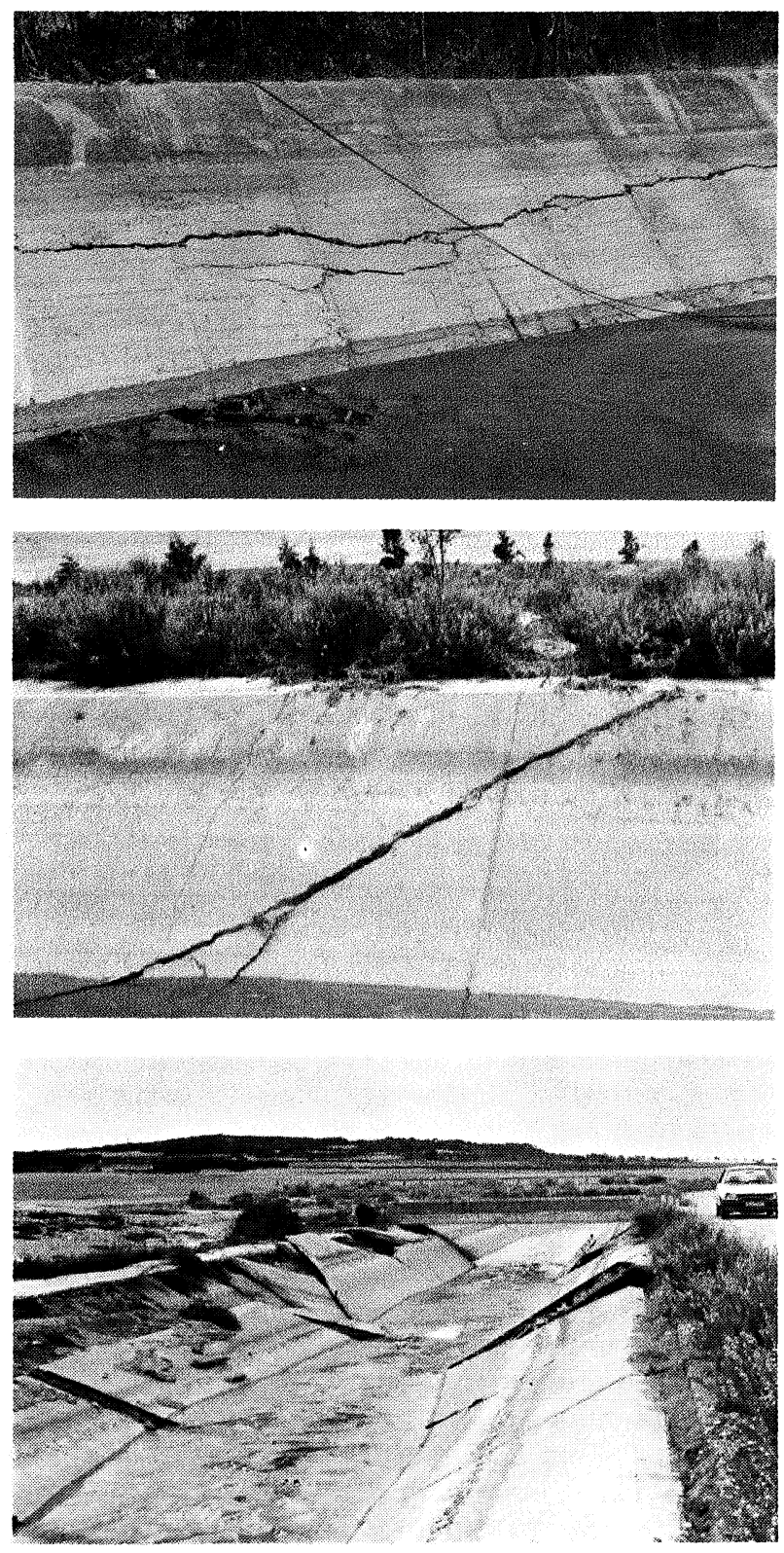

A) Fisuras longitudinales, bien hacia el centro de la solera, bien a una altura determinada de los cajeros que siendo variable, se mantiene prácticamente fija en cada tramo. Este tipo de roturas afecta a grandes longitudes y no se interrumpe en las juntas.

B) Fisuras transversales a la sección, normales al eje en solera y a 450 (típicas de esfuerzo cortante) en cajeros. Afectan a tramos aislados que no suelen pasar en longitud al doble del calado. Presentan discontinuidad o se interrumpen en las juntas de construcción.

C) Movimientos relativos entre losas, con cambio de plano en las juntas. Generalmente estas averias van acompañadas de fisuras irregulares en las losas movidas e, incluso, de la destrucción total o desaparición de losas completas o grandes superficies del revestimiento.

Fig. 1
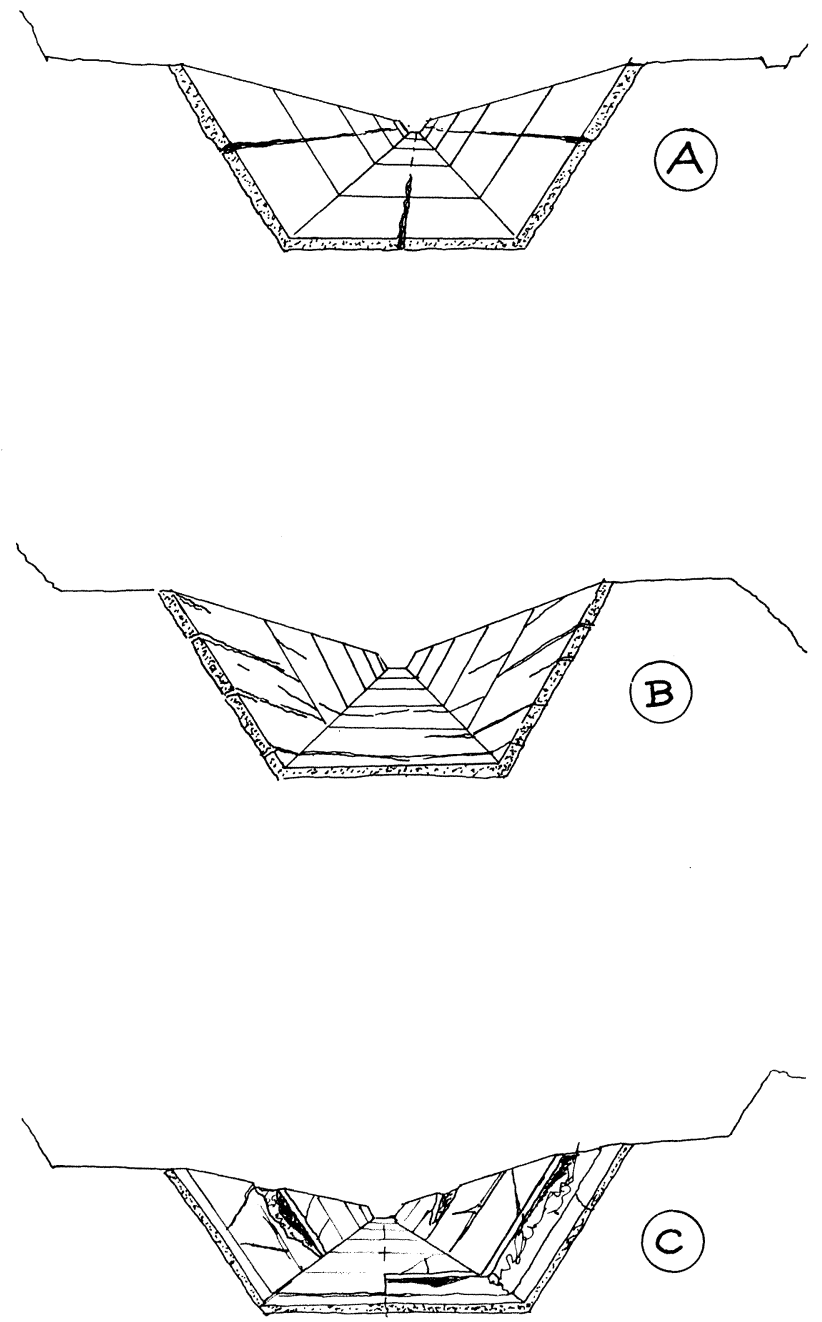

http://informesdelaconstruccion.revistas.csic.es 
Naturalmente, esta clasificación grosera se refiere a las averias observadas en revestimientos bien ejecutados en diseño y calidades, y sobre terrenos con indice de solubilidad aceptables. En los casos de mala ejecución del revestimiento, no hay clasificación posible.

Pero es interesante observar que cada tipo de rotura corresponde a unas circunstancias determinadas del tramo en el que se producen, y solamente se producen cuando existen estas circunstancias. Seguimos hablando de obras bien ejecutadas y, en tales casos, la relación biunivoca es la siguiente:

- Las roturas del grupo A) se producen, con las caracteristicas antes descritas, en tramos en trinchera y nunca en tramos en terraplén o acueducto.

- Las del grupo B) se producen en tramos de transición entre trinchera y terraplén o entre suelos de distinta constitución.

- Las del grupo C) afectan únicamente a revestimientos de placas planas prefabricadas, no inyectados en el trasdós, o a tramos en los que previamente se habia producido una rotura de los tipos A) ० B).

Parece que esta clasificación es válida, prácticamente para todos los canales con independencia de la zona geográfica, tipo de suelo, geometria de la sección u otros parámetros, y es claro que sus motivaciones no pueden darse por incluidas en el campo de los fenómenos de azar.

Existen, evidentemente, un conjunto de circunstancias que producen en el revestimiento los esfuerzos requeridos para su rotura. Tanto los esfuerzos como las circunstancias en las que se producen tienen que obedecer a unas leyes mecánicas generales (puesto que se desarrollan de forma invariable) y son, por lo tanto, previsibles y valorables.

De hecho son sobradamente conocidas en cuanto a su existencia abstracta, pero poco consideradas en cuanto a sus consecuencias prácticas y su cuantificación en cada caso concreto.

Nos vamos a dedicar pues, a reconsiderar lo que ya sabemos, y lo vamos a hacer por los razonamientos simples -los únicos de los que hemos sido capaces- que nos han conducido a una conclusión: "Todo cuerpo sumergido en un liquido, soporta un empuje...". Pues bien, de los tres grupos o tipos de rotura que hemos descrito, podemos decir que tienen en su origen un fenómeno de flotación: las del grupo A) en su totalidad; las del C) en su mayor parte, y las del B) en algunos casos.

Hasta aqui nos hemos referido a las roturas en los canales, posiblemente por ser un tipo de estructura cuyo carácter lineal permite distinguir más claramente los puntos en los que un fenómeno mecánico se produce de los que no lo sufren, pero todas las consideraciones que nos proponemos hacer son válidas para cualquier recipiente enterrado, seal canal, conducto drenante, tubería o depósito.

\section{EL DREN}

El dren: Un concepto claro y una aplicación abusiva. Con demasiada frecuencia se ha esperado del dren lo que el dren no puede dar.

En esencia, el dren no es más que un espacio (lineal o superficial) de suelo con una permeabilidad mayor que la de su entorno.

El prototipo del dren es la zanja, en terreno de baja permeabilidad, relleno de materiales granulares sueltos y que enlanza una zona de suelo saturable de agua con otra filtrante o de cota más baja, de manera que el agua contenida en la primera pueda encontrar salida a la segunda, de forma más rápida que la que se produciria a través del suelo natural.

Desde esta fórmula clásica, hasta las más recientes del tubo-dren o del geotextil, todas ellas buscan la creación de un conducto con mayor permeabilidad que la proporcionada por el terreno natural, al agua contenida en el mismo o recibida por aportaciones puntuales (lluvias, tormentas, subidas del nivel freático), con el fin de evitar la saturación del mismo.

El dren, como cualquier otro conducto, tiene una sección útil, un radio hidráulico y un coeficiente de rugosidad dados. Estas caracteristicas dan un caudal admisible para cada pendiente.

Si el caudal a desaguar es superior a éste, el dren llega a saturación y, a partir de esta situación, entra en carga. También, como en cualquier otro conducto, la circulación del liquido requiere una energía y representa una pérdida de carga proporcional a la distancia recorrida. Esta pérdida de carga viene proporcionada por la pendiente del conducto (el dren en este caso): Cuando ésta no existe o es inferior a la necesaria, la carga viene de un aumento de nivel dentro del dren. Este aumento de nivel llega a alcanzar a la totalidad de la sección y, a partir de este punto, la carga necesaria para la circulación debe ser obtenida de la carga exterior al dren, es decir, de la columna de agua disponible en el exterior; en el propio suelo que se está drenando.

Como, a medida que la longitud del conducto crece, esta presión necesaria para vencer el rozamiento también crece; llega un punto, si la longitud del dren es suficientemente grande, en que toda la presión exterior es necesaria para hacer circular el agua por el interior del dren. Llegada 


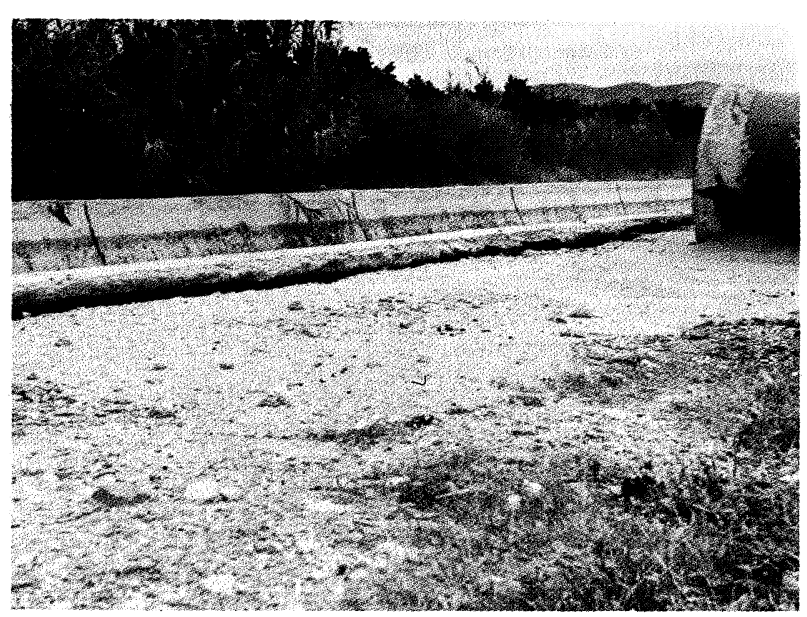

esta situación no se puede aumentar el caudal de desagüe: Se ha alcanzado la saturación de dren y la carga disponible: A partir de esta sección, la presión hidráulica en el terreno se mantiene constante y funciona como si no existiera tal dren. La única utilidad de su presencia es que, si la aportación liquida al suelo es inferior al caudal desaguado en estas circunstancias, la situación se mantendrá, en el tiempo, con una duración inferior a la que hubiera tenido sin la presencia del conducto drenante.

En el esquema adjunto se representan estos estados de tensiones y niveles a lo largo de un dren sumergido en un terreno saturado. En él podemos distinguir:
Tramo I. Desagüe con salida libre. Por encima del nivel freático. No hay aportación de agua. Nivel de lámina prácticamente estable.

Tramo II. El nivel de lámina interno se eleva si la pendiente del dren no es suficiente para vencer el rozamiento interno. Sigue funcionando en lámina libre y a presión atmosférica. Plena eficacia del dren.

Tramo III. El agua ocupa toda la sección del dren. La presión está por encima de la atmosférica y sigue creciendo hasta alcanzar la columna correspondiente al nivel freático exterior al dren. Eficacia decreciente.

Tramo IV. La situación se estabiliza y la presión en el dren es la máxima del nivel freático. Se ha alcanzado la saturación. Dren ineficaz.

Es de señalar que en las circunstancias del final del tramo III y en tramo IV, el dren, no solamente deja de cumplir su misión, sino que al seguir manteniendo una fuerte corriente de agua produce, en los drenes clásicos, un permanente arrastre de finos y erosiones del terreno que terminan por arruinar las obras que se ha pretendido proteger mediante el drenaje.

Estos estados de saturación se han producido con frecuencia en canales, ya que requieren, por su trazado, largos tramos en trinchera o a media ladera. También el fenómeno es frecuente en fondos de depósito de gran superficie.

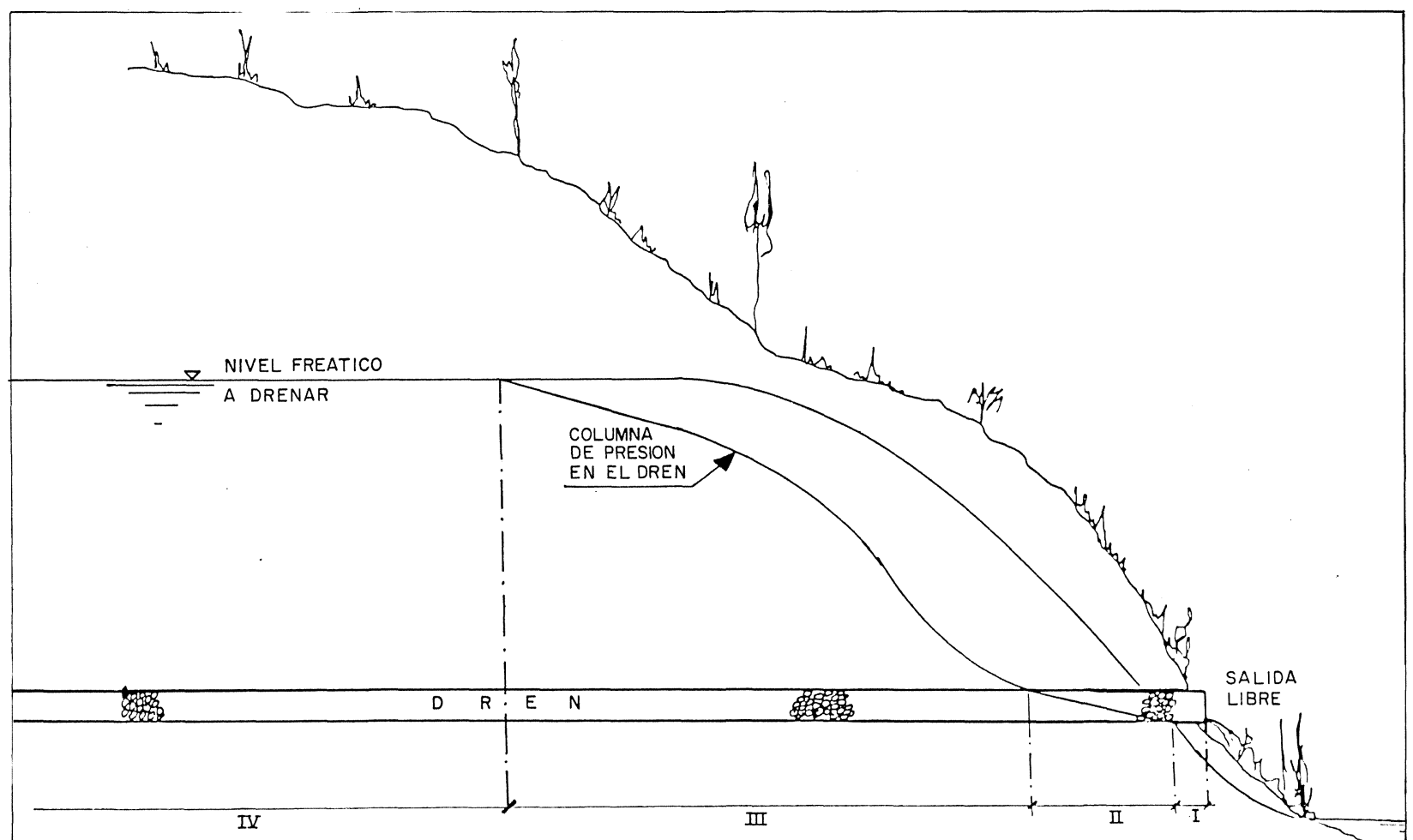


El problema de la erosión de la caja del dren, que acabamos de señalar, tiene un efecto multiplicador, ya que pequeños asientos producidos por esta erosión dan lugar a fisuras que, a su vez, aumentan el caudal en el dren $y$, por ende, la erosión, creando un ciclo que lleva rápidamente a la ruina de la obra.

Salvo diseños muy costosos y cuidados, a base de drenes tubulares con baja rugosidad y capacidad de desagüe muy superior a la teóricamente necesaria, la longitud eficaz de los conductos drenantes es increiblemente baja al poco tiempo de su construcción, lo que en parte es debido a la convergencia de dos procesos contrarios al funcionamiento del dren y que, generalmente, crecen en el tiempo:

Por una parte, el dren tiende a la colmatación y a la disminución de radio hidráulico equivalente. Por otra parte las obras del recipiente (canales o depósitos) sufren deterioros (sellados de juntas que envejecen, fisuras, asientos, etc.) que aumentan sus pérdidas y requieren capacidades crecientes de desagüe.

Esta es la razón por la que, drenes bien dimensionados y que han prestado sus servicios durante años satisfactoriamente, han tenido que ser inyectados y condenados a partir de un determinado momento en el que su función protectora de la obra se ha tornado origen de su ruina.

Hemos comprobado -lo exponemos a título orientativo- que drenes de grava, de $50 \times 50 \mathrm{~cm}$ bajo un canal de $12 \mathrm{~m}^{2}$ de sección, entraban en saturación en distancias de hasta $100 \mathrm{~m}$ de su salida de desagüe, y parece que esta longitud útil se redujo a $30 \mathrm{~m}$ en el transcurso de una tormenta.

Naturalmente, estas longitudes no son válidas en la mayor parte de las trincheras en el trazado de canales que, con frecuencia, miden kilómetros.

Después de lo dicho anteriormente, pudiera parecer que estamos preconizando la supresión del dren bajo canales y depósitos. Nada más lejos de nuestra convicción.

Más bien estamos seguros de que la dinámica destructiva de estas obras solamente se puede evitar mediante un buen drenaje de su entorno. $Y$ esto es lo que proponemos con la solución de auto-dren, a la que nos referimos concretamente al final de estas reconsideraciones sobre ideas ya empleadas en una u otra forma, pero no sistematizadas en su aplicación, ni aun generalizadas.

\section{CUANDO, POR QUE Y COMO SE ROMPEN LOS CANALES}

Es bien conocido, para los ingenieros y personal en contacto con los canales y otros recipientes, el hecho de que las roturas casi nunca se producen en períodos de servicio.

Un canal o depósito raramente tiene problemas cuando está lleno y, si alguno se presenta, siempre se debe a defectos de construcción (mal sellado de juntas; compactación defectuosa de terraplenes; etc.) o a retrasos en su reparación. Con carácter general las roturas de los revestimientos se producen con el recipiente vacio $y$, más frecuentemente, durante el vaciado. $Y$ nos referimos a la rotura, es decir, al momento en el que el revestimiento ve afectada su integridad. Una vez que esto se ha producido, se inician otros procesos destructivos que tienen su origen en la pérdida de impermeabilidad experimentada en el momento de aquella rotura.

Es decir: Si conseguimos que el recipiente soporte el vaciado completo sin sobrepasar sus tensiones admisibles, tendriamos un revestimiento sin problemas, aparte los propios de toda explotación y conservación.

En el epigrafe 1 hemos intentado una clasificación en tres grandes grupos, con caracteristicas propias de las roturas en los canales y que se representan esquemáticamente en la figura 1. Vamos a intentar ahora la justificación lógica de estas roturas y por qué adoptan estas formas tipicas.

Es hecho comprobado, y en la práctica inevitable, que en el entorno de un revestimiento de canal o depósito hay una presencia de agua que proviene de fugas por las juntas, pequeñas fisuras, poros, etc.

El grado de saturación que este agua produce en el terreno, depende del caudal aportado por estas fugas y de la permeabilidad del mismo.

También, en ciertos momentos, las aguas procedentes de las lluvias, otros cauces de agua próximos, etc. representan aportaciones adicionales y frenan la evacuación de las primeras.

En todos los tramos averiados, en los que hemos realizado catas en los caminos de servicio hasta alcanzar el trasdós del revestimiento, hemos podido comprobar que existe un nivel freático artificial que, en el trasdós del cajero, está muy próximo al del interior de la sección, y baja rápidamente al alejarse de éste. En algunos casos de trincheras, en laderas con fuerte escorrentía, este nivel supera al de explotación del canal debido a que el propio cajero actúa como una pantalla de retención de la escorrentia del suelo. 
Estas condiciones se representan en la Fig. 2, y las leyes de empujes y esfuerzos que esta masa de agua ejerce sobre el revestimiento quedan esquematizadas en las sucesivas figuras $3,4,5,6$.

Si a estas leyes se suman las debidas al peso propio del revestimiento y las debidas a la carga de agua en el interior del canal, siempre tenemos

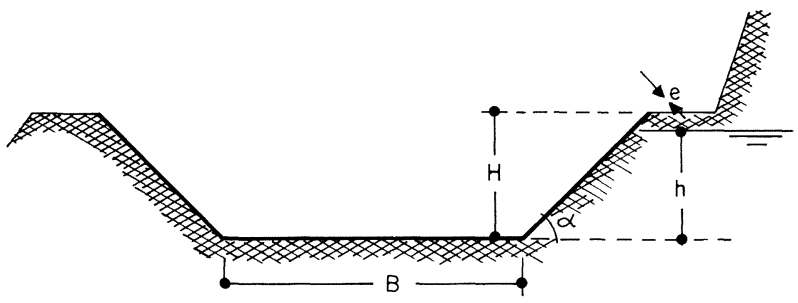

Fig. 2. - Situacion de canal vacıo y terreno saturado.

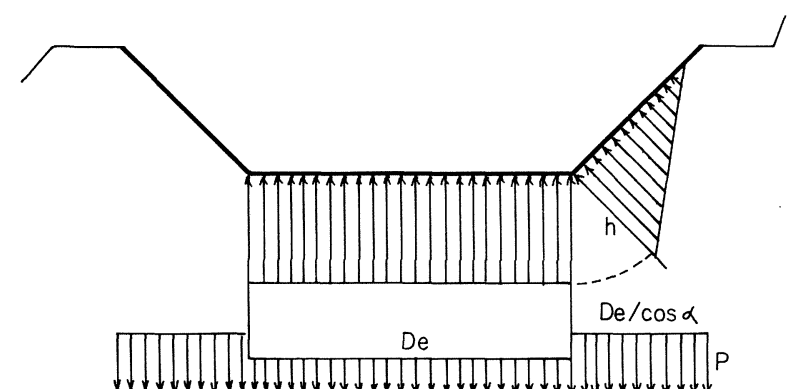

Fig. 3. - Ley de presiones hidrostaticas sobre paramento exterior. e: Espesor del revestimiento.

$D=$ Peso especifico del material.

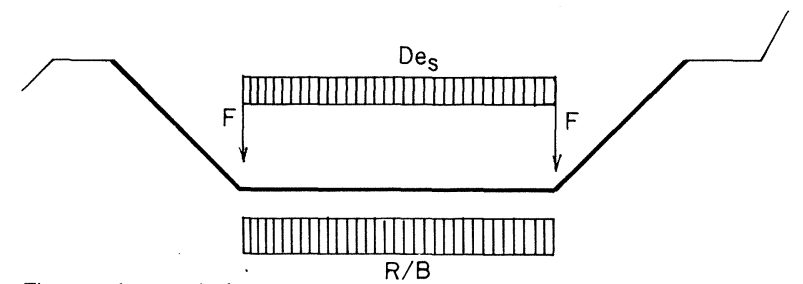

Fig. 4. - Leyes de fuerzas

$P=$ Peso propio del revestimiento.

$E=$ Empujes por sub-presión.

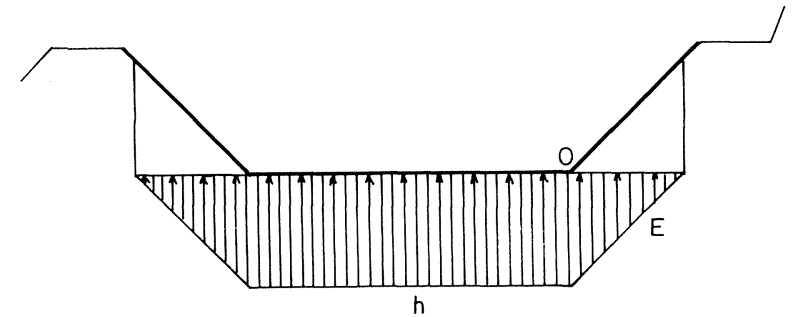

Fig. 5. - Fuerzas resultantes sobre solera.

$F=$ Acción del peso de los cajeros sobre solera. $R=$ Resultante del peso propio.

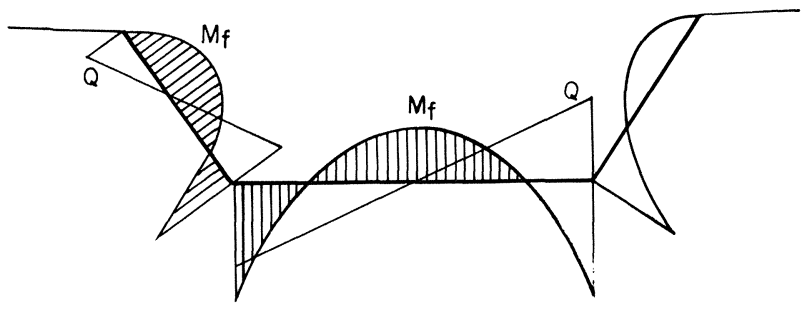

Fig. 6. - Leyes de esfuerzos resultantes en el revestimiento. una resultante en el sentido de apoyar el revestimiento sobre el terreno, con valores iguales o inferiores a los de cálculo de la sección.

Pero supongamos que se inicia un vaciado de forma que la velocidad de descenso del nivel interior es mayor que la del nivel freático exterior (lo que sucede habitualmente). En tal caso, empiezan a dominar los empujes exteriores y, a partir de la situación en que la diferencia de empujes (exterior e interior) supera al peso propio del revestimiento, se inicia la flotación de la sección.

Esto no es una metáfora: realmente, el revestimiento se comporta como el casco de un barco e inicial el despegue del terreno.

En la secuencia de la Fig. 7 se representan las evoluciones de los empujes soportados por la sección en los casos que podriamos denominar como canales estrechos y anchos; es decir, cuando las relaciones aproximadas (el fenómeno tensional depende también de la calidad del material de revestimiento y de sus espesores) de calado a anchura son mayores o menores que un valor situado entre 1 y $1 / 2$

El proceso mecánico que se pone en acción con este inicio de flotación es:

a) Si la sección es profunda (H/B $>1$ ) la flotación se produce realmente; los cajeros quedan en voladizo y cuando la tensión de tracción en la cara interna alcanza su valor de rotura, el cajero quiebra a lo largo de una línea (la determinada por esta tensión límite) sensiblemente paralela al eje del canal.

b) Si la sección es ancha $(H / B<1 / 2)$ la línea critica se alcanza antes en solera que en cajeros y aparece una fisura situada en las proximidades del centro de la misma.

\section{Estos son los casos de rotura típica del tipo A.}

Naturalmente, estas roturas iniciales ponen en marcha un proceso de segundo orden: A partir de estas roturas el revestimiento pierde su estanquidad; en los llenados sucesivos las pérdidas de agua son importantes; el canal ya no es capaz de flotar (el casco del barco está destrozado); el terreno soporte se fluidifica y pierde finos que entran en el canal, y en un corto número de ciclos de llenado y vaciado se han cambiado de tal forma, las hipótesis del proyecto, que el canal se convierte en un cauce en adaptación permanente a sus nuevas circunstancias. El proceso de degradación conduce a las averias del grupo $\mathrm{C}$ como veremos más adelante.

En ciertas explotaciones de canales, con un enfoque práctico de su temática se ha optado por no sellar las juntas y preferir los revestimientos de baja calidad y más permeables. Se ha captado, empíricamente, que en tales revestimientos no se les plantean los problemas de la flotación. 


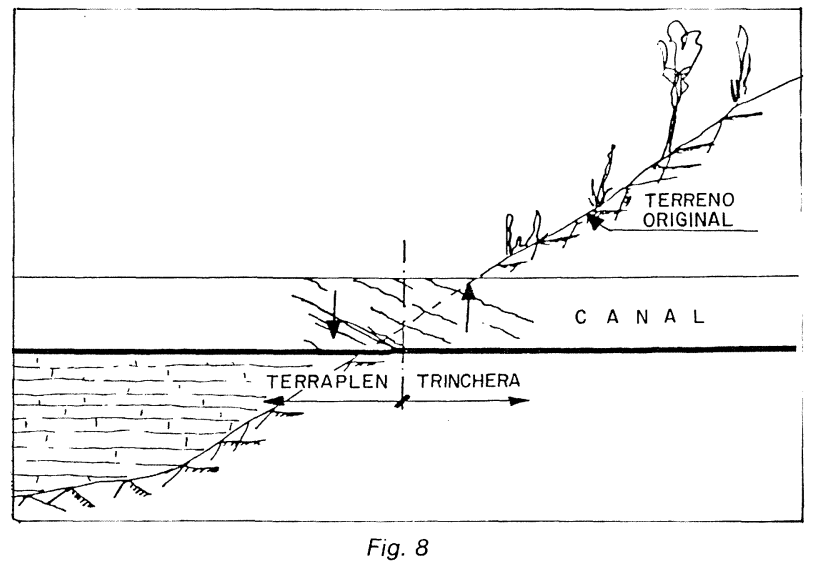

Si el terreno soporte tiene buena cohesión y no es muy permeable llegan a obtener un cauce formado por el terreno natural mejorado por el revestimiento en cuanto a rugosidad y limitación de la vegetación. Es una solución imperfecta pero, a veces, mejor que las obtenidas con revestimientos impermeables sujetos al fenómeno de la flotación. $Y$ es claro que un revestimiento permeable no puede flotar. Puede hacer sin embargo disminuir, de forma importante, los efectos de la erosión.

Pasemos a considerar las roturas del tipo $\mathrm{B}$, con fisuras en cajeros a 450 .

Estas roturas se producen, como hemos dicho anteriormente, en las zonas de transición entre trinchera y terraplén o entre terrenos de distinta permeabilidad.

En la figura 8 se presenta el esquema caracteristico de la situación en sección longitudinal.

Ante tal esquema la primera tentación es achacar la rotura al asiento del terraplén, máxime cuando una consecuencia clásica de ese tipo de roturas es la descomposición y arrastre de una parte importante de aquél.

Sin embargo este tipo de fisuras es relativamente frecuente en casos de terraplenados perfectamente ejecutados y en los que se ha podido comprobar que no se han producido asientos diferenciales con el terreno original.

En otros casos, este tipo de roturas se ha producido sin existencia de terraplenes: Simplemente existia una transición entre un terreno impermeable y otro permeable, ambos originarios. Comprobaciones más precisas que las puramente intuitivas, demuestran que no ha bajado el tramo en terraplén, sino que ha subido el tramo en trinchera. $Y$, como siempre, la rotura se ha producido en fase de vaciado o en el transcurso de una tormenta con el canal vacio.

Los responsables de la explotación de canales saben que el indice de ventas sobre tramos en terraplén es incomparablemente menor que el que se produce en los tramos en trinchera, y es prácticamente nula en los tramos en acueducto.

La razón es obvia: Los acueductos no tienen terreno circundante; los terraplenes son de gran permeabilidad (materiales granulares); y las trincheras son de terreno natural, colmatado y de permeabilidad generalmente baja.

Solamente puede haber flotación cuando la obra puede estar sumergida.

No intentamos, con todo ello, mantener que las roturas del tipo $\mathrm{B}$ tengan siempre su origen en la flotación del tramo en trinchera: Es evidente que, en algunos casos, se producen por asientos diferenciales. Solamente señalamos que este tipo de roturas puede ser producido, también, por fenómenos de subpresión.

En estos ćasos la rotura suele continuarse en otra del tipo A. Finalmente, vamos a considerar el grupo C de roturas, señalado anteriormente, es decir, las que, sin guardar ninguna caracteristica morfológica concreta, se traducen en la destrucción de zonas importantes o tramos enteros del revestimiento.

Se producen tras un proceso de tres fases:

1. a Aumento de la permeabilidad del revestimiento en proporciones suficientes para afectar a las caracteristicas resistentes o composición del terreno, lo que puede tener su origen en roturas de los grupos A ó B, o fallos en los sellados de juntas.

2. a Pérdida de apoyo en el terreno y asientos con movimiento relativo entre losas resultantes de roturas anteriores por fisuración. Estos movimientos relativos dan lugar a superficies escalonadas que impiden el régimen laminar y crean turbulencias que siguen el proceso de erosión del terreno en el trasdós. En un proceso acelerado, esta destrucción del suelo provoca nuevos movimientos y nuevas lineas de rotura, al tiempo que se crean superficies frontales a la corriente.

3.a En el cuarteo sucesivo, se llega a la situación en que las losas resultantes pierden su estabilidad ante los empujes dinámicos de la corriente y los trozos que llegan a tal situación de solicitaciones son desplazados y arrastrados.

Los huecos resultantes son de nuevo atacados por los fenómenos de erosión y la presión dinámica de la corriente actúa en el trasdós del revestimiento restante continuando el proceso de forma imparable.

Sin necesidad de roturas previas, este tipo de averias es frecuente en revestimientos realiza- 

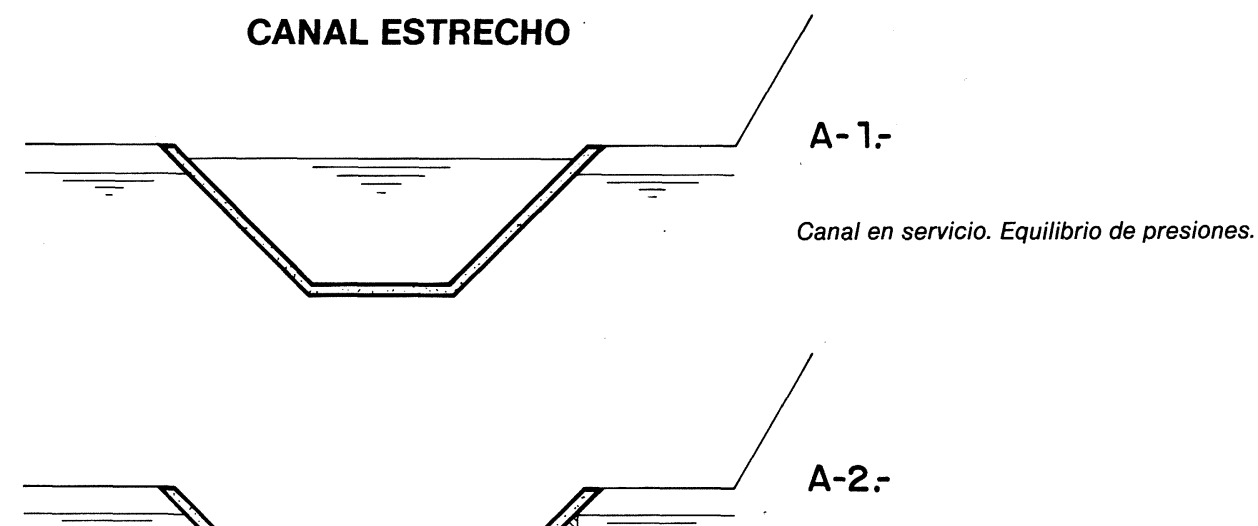

Vaciado rápido. Se inician las subpresiones. No se llega a la flotación.
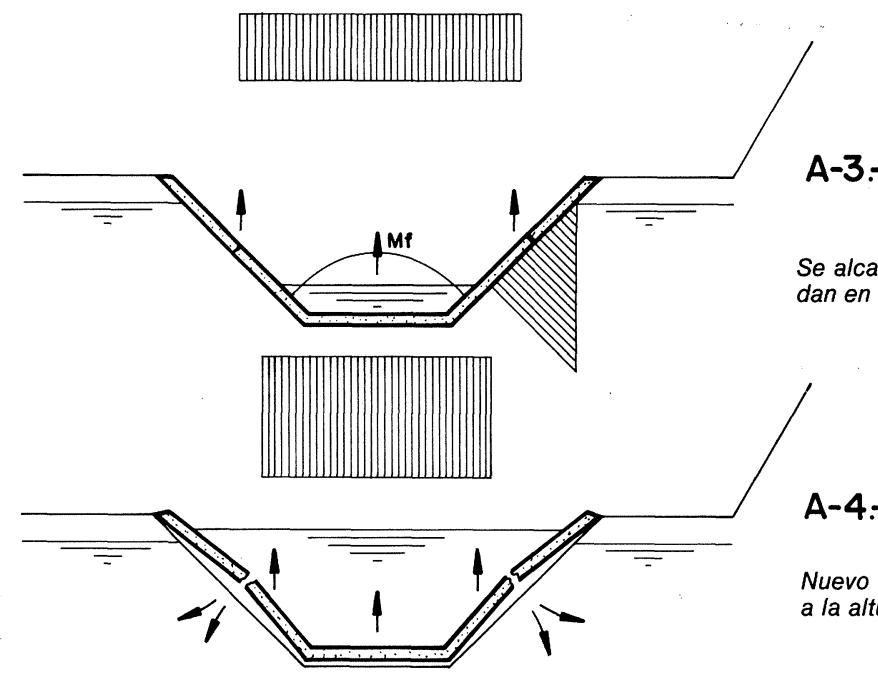

Se alcanza el empuje de flotación. No rompe la solera. Los cajeros quedan en voladizo y quiebran por la sección crítica.

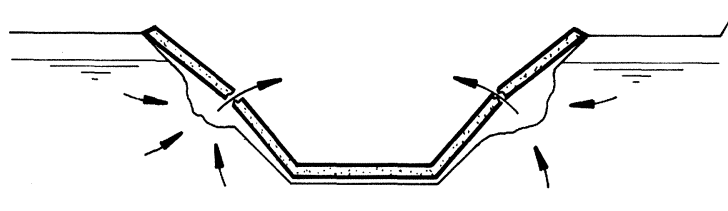

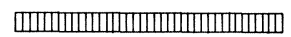

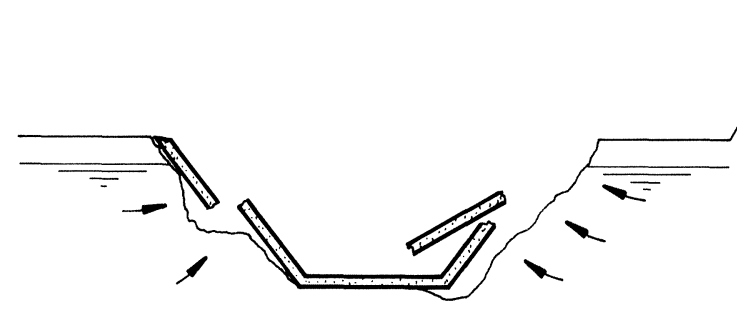

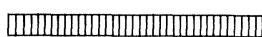

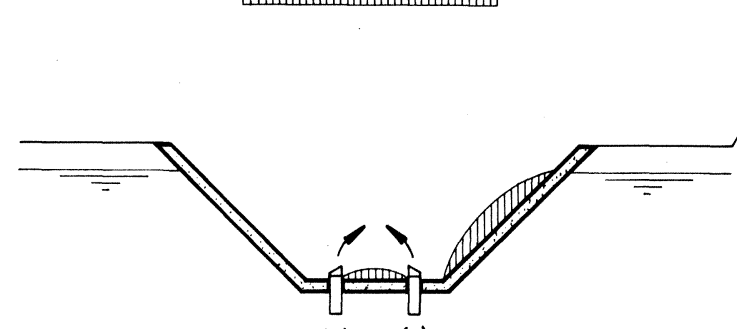

$1>1$

\section{A-6.-}

La repetición del ciclo descalza gravemente los cajeros y la solera, con desprendimiento de la zona de cajero superior a la fisura. Entra en estado de deterioro debido a la erosión. Colabora la subpresión interna del suelo que se va fluidificando. jeros drena la subpresión con arrastre de suelo. Los cajeros empiezan a quedar sin trasdós. 


\section{CANAL ANCHO}
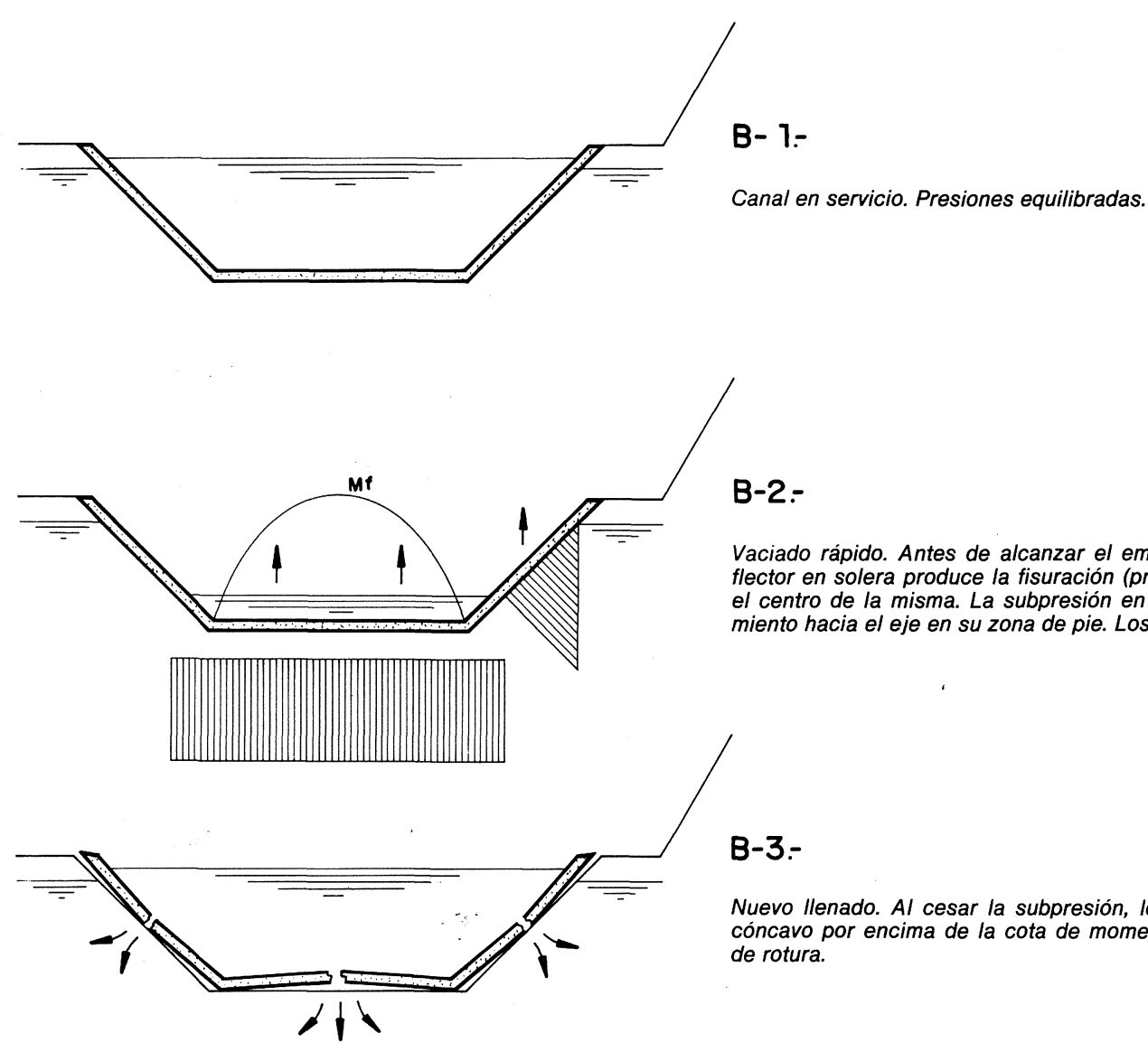

\section{B-2:-}

Vaciado rápido. Antes de alcanzar el empuje de flotación, el momento flector en solera produce la fisuración (próxima al eje). Sube la cota en el centro de la misma. La subpresión en cajeros produce su desplazamiento hacia el eje en su zona de pie. Los cajeros quedan en flexión.
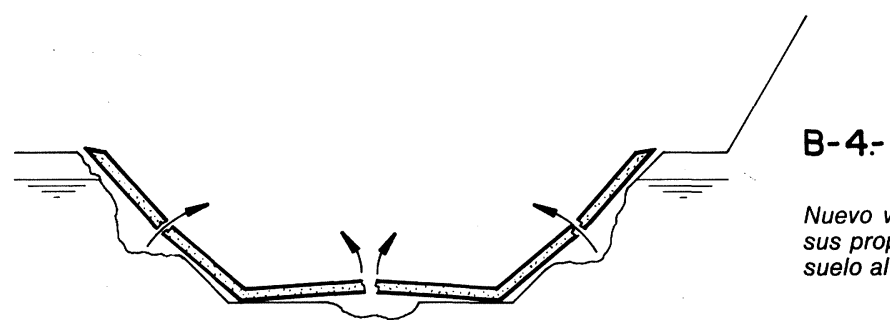

Nuevo vaciado. Apenas hay subpresiones. El revestimiento ha buscado sus propios drenes por roturas de los paramentos y solera. Arrastre de suelo al interior de la sección. Descalce de cajeros y solera.

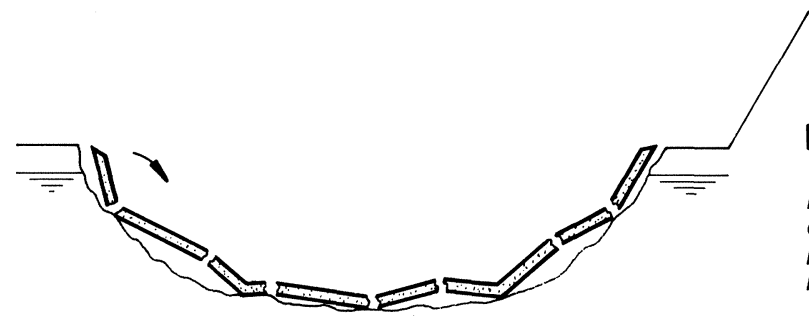

\section{B-5-}

La repetición del ciclo lleva a nuevas erosiones y nuevos descalces de trasdós hasta cuartear toda la sección por flexiones sucesivas. El proceso es de crecimiento geometrico. La ruina del revestimiento imparable.

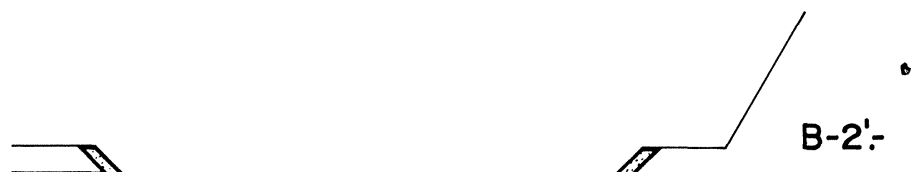

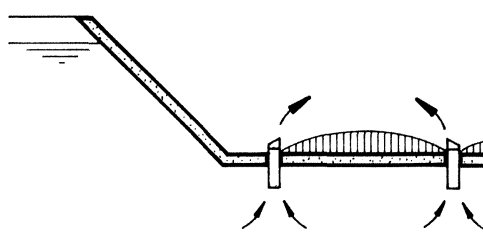

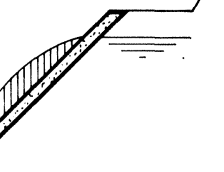

Se han

tado depresores de fondo, por lo que el agua exterior se al canal creando puntos de subpresión cero. No se produce la subpresión se mantiene tan baja como sea preciso. Tanto la obra como sus posibles reparaciones se mantienen en los limites tensionales admisibles. 
dos con losas planas prefabricadas y no inyectadas en trasdós. Ello se debe a varias razones: En primer lugar, las losas prefabricadas presentan, inevitablemente, un apoyo imperfecto e incompleto sobre el terreno, especialmente en solera, lo que da lugar a movimientos posteriores al montaje por pequeños o grandes asientos en las zonas de apoyo. Estos, tienen como consecuencia una pérdida de estanquidad en las juntas asi como resaltos en las mismas, con el inicio de los procesos descritos anteriormente.

Por otra parte, los elementos prefabricados, ejecutados con hormigones de alta calidad, permiten espesores menores $y$, consecuentemente, menores pesos por unidad de superficie y mayor movilidad.

Todo ello hace que, el primer esfuerzo de flotación, altere fácilmente el esquema teórico del revestimiento y se produzcan averias de tipo $\mathrm{C}$, directamente y sin pasar por estados intermedios.

\section{EL AUTO-DREN}

De todo lo expuesto anteriormente se puede hacer el resumen siguiente:

- No es posible mantener un canal en buenas condiciones de conservación, mientras quede sometido a empujes de flotación.

- La única forma de que no se produzcan estos empujes es evitar la presencia de agua en saturación en el terreno circundante.

- Si el propio terreno no tiene la capacidad drenante suficiente para garantizar la imposibilidad de saturación, la solución del dren clásico solamente es válida para tramos cortos en trinchera. Los puntos distantes más de $100 \mathrm{~m}$, como cifra orientativa de los desagües del dren, son puntos con riesgo de subpresiones.

- En tramos más cortos, en los que el dren pueda ser eficaz, éste crea un riesgo de erosión de la sub-base y asientos en la obra.

- Impidiendo la subpresión, se asegura la inmovilidad vertical y la correcta transmisión de las cargas al terreno. Estas condiciones permiten realizar, con éxito, una buena solución de estanquidad en juntas.

- Un revestimiento estable y estanco no sufre, en principio, ningún esfuerzo imprevisto y debe obedecer a las hipótesis resistentes del proyecto.

- Resulta obvio que cualquier reparación que intente la reposición de las condiciones de impermeabilidad, en tramos que hayan sufrido los efectos descritos, está destinada a nueva rotura en la primera ocasión en que las causas de la averia original vuelvan a presentarse.
Analizada la situación a la luz del fenómeno real, tal como se ha expuesto, llegamos a la conclusión, como solución práctica, de la necesidad de disponer de un esquema de drenaje que cumpla las condiciones siguientes:

a) Debe asegurar que en ninguna sección se pueda producir una fuerza por subpresión superior a la que la sección puede soportar.

En ningún punto de las secciones de hormigón, deberán tenerse columnas de agua de altura superior a 2,2-2,3 veces el espesor de la solera, pues a partir de este punto se iniciaria la elevación de ésta y esfuerzos de flexión.

b) Debe poder ejercer su función con independencia de su longitud.

c) No se requiere su funcionamiento en tanto la presión hidráulica en el interior supere a la exterior. Incluso presentaría ventajas para la estabilidad de la obra la evitación de corrientes y movimientos de agua en el seno del terreno, mientras la presencia de agua en el mismo no represente amenaza de empujes inadmisibles.

d) Seria deseable la obtención de una solución aplicable a los canales o depósitos ya construidos y averiados para hacer posible su reparación y conservación posterior, con garantia de permanencia.

e) Debe originar unos costes de construcción más conservación inferiores a los de una obra en avería quasi-permanente.

Analizados estos condicionantes en su conjunto, proponemos la solución que hemos denominado "Auto-Dren", ya que es la propia caja del canal, o el propio depósito en su caso, los que sirven para recoger el agua contenida en el terreno, de forma que ésta pasa del suelo al interior de la sección, a medida que ello es necesario, para el deseado equilibrio de presiones entre las dos caras de los paramentos.

En efecto, la condición b) exige la posibilidad de desagüe en toda la longitud y con independencia de cuál sea ésta.

Si queremos que esta condición se pueda cumplir en el caso frecuente de canales que discurren en trincheras en la totalidad de su traza o, en cualquier caso, a lo largo de varios kilómetros, estamos obligados a optar entre la disposición de puntos de recogida a cota inferior a la del canal, mediante pozos laterales y elevación posterior por bombeo, o a utilizar la propia sección del canal como conducto drenante, ya que la solera de éste es la única cota superficial practicable y accesible que podemos disponer por debajo del nivel freático máximo admisible, en el entorno de la obra. 
Naturalmente, la primera solución de drenaje por bombeo, válida para casos muy concretos con energía disponible (fondos de depósitos en zonas industrializadas, p.e.) sería impensable a lo largo de la traza de un canal, en los casos más frecuentes.

Por otra parte, la utilización del propio cauce como desagüe proporciona importantes ventajas, como veremos a continuación.

En un terreno ideal, totalmente impermeable e inalterable por la saturación superficial en sus caracteristicas reológicas, bastaría con dar al revestimiento una permeabilidad suficiente entre sus caras. Por ejemplo juntas abiertas o taladros en solera.

Pero es el caso que los suelos no gozan de estas propiedades, en la medida suficiente, a fin de evitar que tal solución implique pérdidas importantes de agua, y unas consecuencias de deterioro por asientos de la obra, erosión, etc.

La solución Auto-Dren está fundada en limitar este efecto de permeabilidad a la dirección exterior-interior, manteniendo la estanquidad en la opuesta: Un dispositivo de paso anti-retorno permite asegurar la estanquidad del revestimiento en tanto la presión interior supera a la exterior.

Cuando esta relación de presiones se invierte creando una situación inicial de posibilidad de flotación, se produce, de forma automática, la apertura del mecanismo anti-retorno con paso del agua del terreno hacia el interior de la sección, haciendo imposible el estado de presiones

\section{CANAL CON DREN INFERIOR}

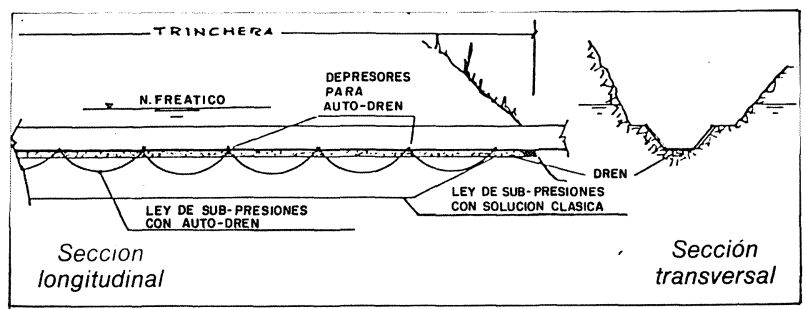

que, indefectiblemente, conduce a la rotura del revestimiento.

El canal, o en su caso el depósito, sirve asi de conducto drenante de su propio entorno.

En los casos en que se disponga de un dren previo, bajo solera, cuya longitud sea excesiva o cuya sección sea insuficiente, ya hemos vistó cómo se produce la puesta en carga y la inefectividad en cuanto a subpresiones se refiere.

La aplicación del mecanismo de Auto-Dren se realiza mediante taladro de la solera y conexión del conducto drenante con el interior de la sección a través del depresor, y crea tantos puntos de presión nula como sea preciso para garantizar valores admisibles de la subpresión, o bien para asegurar presiones cero en todo el conducto.

Lo que realmente estamos obteniendo es la descomposición de un dren de gran longitud en tantos tramos con desagües externos, como deseemos.

Con esta solución, resulta conveniente el taponamiento de los antiguos desagües extremos. En efecto: Si sustituimos el dren por un conducto permeable y ciego, vamos a conseguir que en este conducto haya circulación de agua solamente en los momentos en que el agua es perjudicial para la obra, es decir, cuando baja el nivel $y$, por lo tanto, la presión en el interior de la sección. Se evitan asi dos efectos nocivos del funcionamiento del dren: Pérdidas de agua innecesarias y erosiones por pérdida de finos en el suelo.

Cuando no existe conducto drenante previo, la puesta en acción del depresor se realiza en dos etapas:

1.a En función de la permeabilidad del suelo, se crea una zona en el entorno del depresor en la que el agua pasa a la sección sin producir movimiento alguno en el revestimiento, y en la que las presiones requeridas son infe-

2. CANAL SIN CONDUCTO DRENANTE

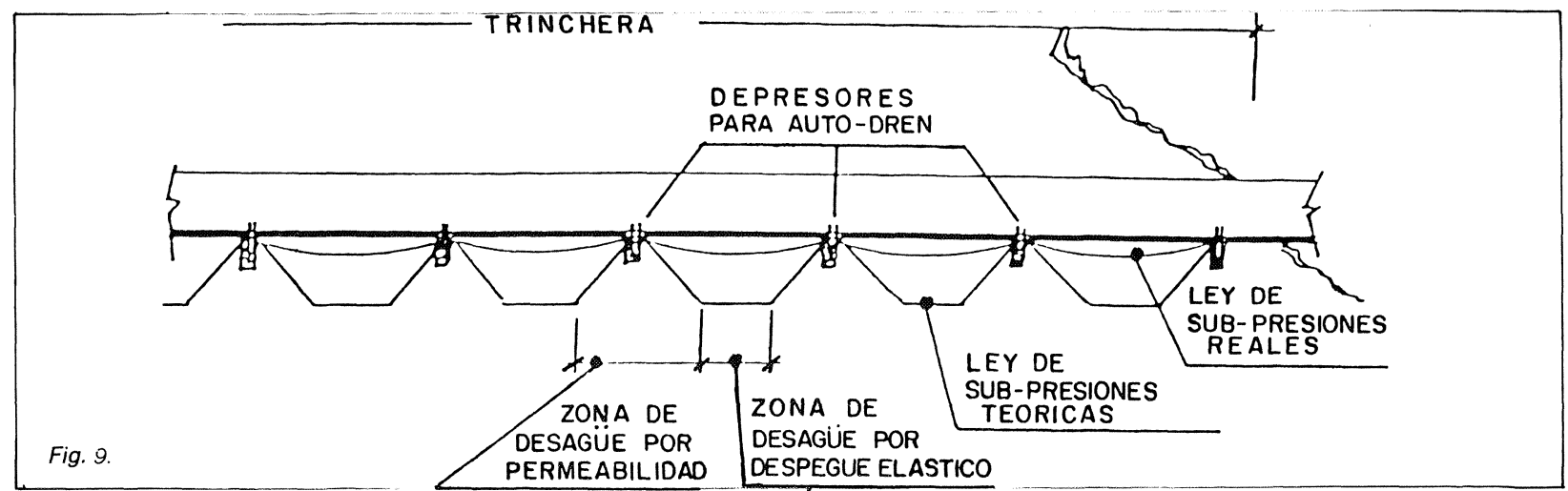




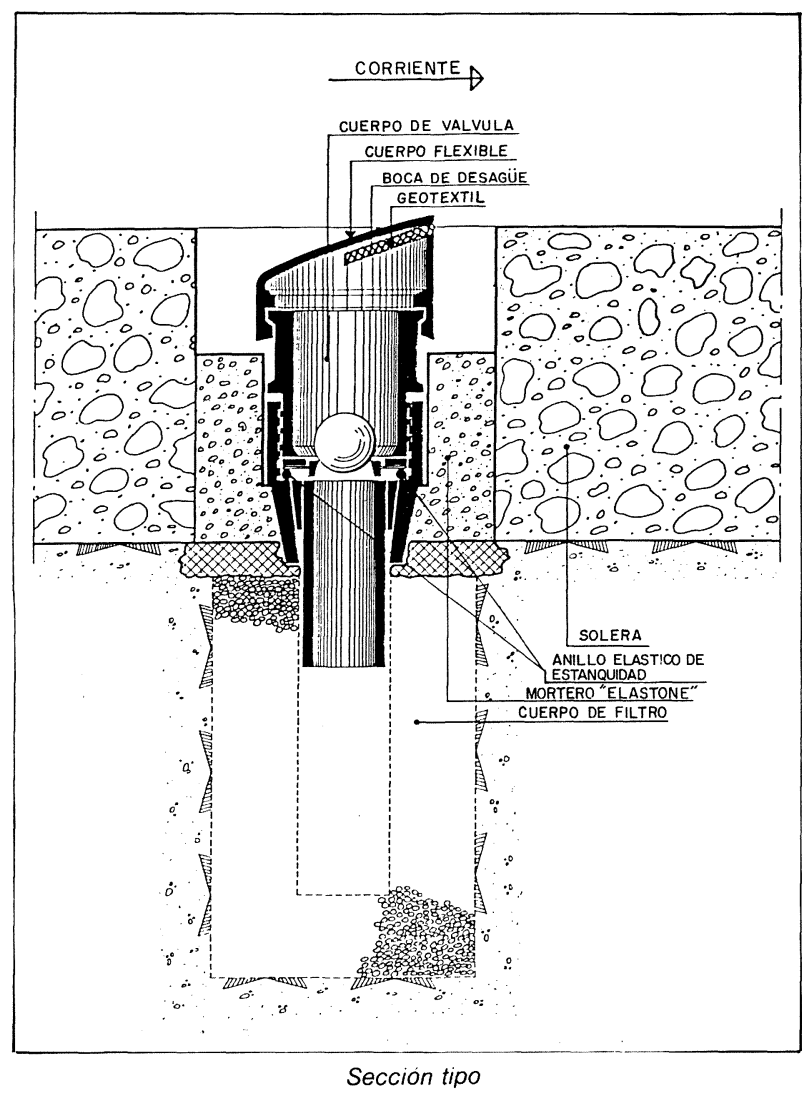

riores a las creadas por el peso de la sección.

2.a A partir de esta zona que podriamos denominar de desagüe por permeabilidad, se llegaria, en teoria, a presiones de flotación: El revestimiento iniciaria su despegue del terreno pero, automáticamente, este movimiento equivaldria a un aumento de la zona de permeabilidad; el agua encuentra desagüe inmediato, baja la presión y el despegue se hace imposible. El revestimiento no sobrepasa su periodo elástico si los puntos de desagüe quedan situados a distancias tales que la subpresión no pueda alcanzar, de forma permanente, el valor de flotación.
Ello requiere, pues, un estudio de cada caso, en función de las caracteristicas de la sección y de la permeabilidad del suelo. En estos casos es recomendable, como norma general, la ampliación de la zona de desagüe por permeabilidad, mediante la creación de sondas drenantes bajo el depresor. En la figura 9 se expresa, gráficamente, el esquema de funcionamiento del Auto-Dren en los dos casos descritos.

Hemos comprobado que el sistema Auto-Dren, de gran simplicidad en su esquema de funcionamiento, requiere especial atención en varios aspectos para la optimización de sus rendimientos y para su explotación sin problemas de conservación, que se resumen en los puntos siguientes:

- Estudio detallado de emplazamientos y distancias entre depresores.

- Utilización de materiales no alterables por el agua o la humedad, muy especialmente en el cuerpo de válvula.

- Adecuado dimensionamiento del propio depresor y de su sonda drenante, cuya porosidad debe condicionarse a la constitución del suelo.

- Corrección en el montaje que garantice la estanquidad y la facilidad en la reposición del cuerpo de válvula, en su caso.

- Diseño del cuerpo del depresor para evitar eficazmente la entrada en su interior de cuerpos sólidos, algas, etc. así como la formación de las mismas.

La figura 10 muestra la sección de uno de los diseños que hemos empleado con resultado satisfactorio en todos estos aspectos. Deseariamos que la exposición que antecede pueda ser útil para evitar una parte importante de las averías de canales, conducciones y depósitos enterrados. Tanto los problemas de explotación que se derivan de las roturas en este tipo de obras, como el coste a que dan lugar justifican, ampliamente, el interés y la esperanza que ponemos en que el Auto-Dren sea, realmente, una solución. 